\title{
Correction
}

\section{Correction to: Increasing targeting scope of adenosine base editors in mouse and rat embryos through fusion of TadA deaminase with Cas9 variants}

\author{
Lei Yang ${ }^{1}$, Xiaohui Zhang ${ }^{1}$, Liren Wang ${ }^{1}$, Shuming Yin ${ }^{1}$, Biyun Zhu', Ling Xie', Qiuhui Duan ${ }^{1}$, \\ Huiqiong $\mathrm{Hu}^{1,2}$, Rui Zheng ${ }^{3}$, Yu Wei ${ }^{1}$, Liangyue Peng ${ }^{1,4}$, Honghui Han ${ }^{5}$, Jiqin Zhang ${ }^{1}$, Wenjuan Qiu ${ }^{3}$, \\ Hongquan Geng ${ }^{3}$, Stefan Siwko ${ }^{6}$, Xueli Zhang ${ }^{1,2}$, Mingyao $\mathrm{Liu}^{1,6}$, Dali $\mathrm{Li}^{1 \bowtie}$ \\ ${ }^{1}$ East China Normal University and Shanghai Fengxian District Central Hospital Joint Center for Translational Medicine, \\ Shanghai Key Laboratory of Regulatory Biology, School of Life Sciences, East China Normal University, Shanghai 200241, \\ China \\ ${ }^{2}$ Fengxian Hospital Affiliated to Southern Medical University, Shanghai 201499, China \\ ${ }^{3}$ Xinhua Hospital, Shanghai Jiao Tong University School of Medicine, Shanghai 200092, China \\ ${ }^{4}$ School of Life Sciences, Hunan Normal University, Changsha 410081, China \\ ${ }^{5}$ Bioray Laboratories Inc., Shanghai 200241, China \\ ${ }^{6}$ Department of Molecular and Cellular Medicine, The Institute of Biosciences and Technology, Texas AM University Health \\ Science Center, Houston, TX 77030, USA \\ $\triangle$ Correspondence: dlli@bio.ecnu.edu.cn (D. Li)
}

CORRECTION TO: PROTEIN CELL 2018, 9(9):814-819

HTTPS://DOI.ORG/10.1007/S13238-018-0568-X

In the original publication the grant number is incorrectly published. The correct grant number should be read as "17140901600". The corrected contents are provided in this correction article. This work was partially supported by grants from the National Natural Science Foundation of China (Nos. 81670470 and 81600149), a grant from the Shanghai Municipal Commission for Science and Technology (17140901600, 18411953500 and 15JC1400201) and a grant from National Key Research and Development Program (2016YFC0905100).

\section{OPEN ACCESS}

This article is distributed under the terms of the Creative Commons Attribution 4.0 International License (http://creativecommons.org/ licenses/by/4.0/), which permits unrestricted use, distribution, and reproduction in any medium, provided you give appropriate credit to the original author(s) and the source, provide a link to the Creative Commons license, and indicate if changes were made.

The original article can be found online at https://doi.org/10.1007/ s13238-018-0568-x. 